\title{
How to demix Alzheimer-type and PSP- type tau lesions out of their mixture -hybrid approach to dissect comorbidity-
}

\author{
Momoko Ebashi ${ }^{1,2}$, Yoshinori Ito ${ }^{1,3}$, Miho Uematsu1, Ayako Nakamura' ${ }^{1}$ Katsuiku Hirokawa ${ }^{4}$, Satoshi Kamei ${ }^{2}$ and \\ Toshiki Uchihara ${ }^{1,5^{*}}$
}

\begin{abstract}
Neurofibrillary tangles (NFTs), are shared between progressive supranuclear palsy (PSP) and Alzheimer disease (AD). Histological distinction of PSP and AD is possible based on the distribution of NFTs. However, neuropathologists may encounter diagnostic difficulty with comorbidity of PSP and AD. In this study, we tried to circumvent this difficulty by analyzing five autopsied brains harboring both PSP and AD pathology. Tau-positive lesions were sorted based on their cell type (neuron versus glia), and tau isoforms: three-repeat (3R) versus four-repeat (4R) tau. 16 regions were selected to map these lesions throughout the brain. 4R-tau lesions were present in all areas examined. Among them, 3R-tau lesions were absent in some areas. These $4 R$ selective (4R+/3R-) areas dictate prototypic distribution of PSP, not usually found in AD, such as pontine nucleus, red nucleus, inferior olivary nucleus, dentate nucleus, globus pallidus and putamen, each contained both glial and neuronal lesions. In contrast, additional 3R-tau lesions were found in hippocampal formation to neocortex, where 3R immunoreactivity (IR) was predominant over the 4R counterpart mainly in neurons as found in AD but not in PSP. Although tau lesions in central grey matter, substantia nigra and locus coeruleus are found in both AD and PSP, 4R-selectivity with glial component suggests PSP origin. Even if the presence of $3 \mathrm{R} I R$ in these areas suggests AD pathology, it does not exclude the involvement of PSP-type lesion because distinction of $4 R$ IR into PSP or AD is not yet possible. Further demixing may be possible if biochemical difference of $4 R$ tau between PSP and AD is identified.
\end{abstract}

Keywords: Tau cytopathogy, AD, PSP, Comorbiditiy, Isoform, Glia

\section{Introduction}

Alzheimer disease $(\mathrm{AD})$ and progressive supranuclear palsy (PSP) are characterized by deposition of tau in the brain. Initially, histological definitions were based on the disease-specific distribution of argyrophilic neurofibrillary tangles (NFTs), which are quite distinct between AD [1] and PSP [31]. Another histological hallmark of PSP is tuft-shaped astrocytes (TAs) [15], which are essentially not found in pure AD. Because clinicopathological spectra of PSP and of AD are still expanding [10, 30], accurate clinical diagnosis is more and more complex and difficult.

\footnotetext{
* Correspondence: uchihara-ts@igakuken.or.jp

${ }^{1}$ Laboratory of Structural Neuropathology, Tokyo Metropolitan Institute of Medical Science, Tokyo, Japan

${ }^{5}$ Neurology Clinic with Neuromorphomics laboratory, Nitobe-Memorial

Nakano General Hospital, Tokyo, Japan

Full list of author information is available at the end of the article
}

This diagnostic difficulty is much enhanced in aged population, where cormorbid pathologies, such as AD or Lewy pathology, may be encountered. [10, 30]. Such comorbidity is challenging to neuropathologists; how to discriminate different types of pathology in the same brain. This is particularly problematic when a brain harbors AD-type pathology and PSP-type pathology because both are characterized by tau deposits $[3,11]$. Furthermore, it is not yet known whether these two types of pathology are independent or mutually related. In this study, we selected autopsy samples carrying histological diagnoses of both $\mathrm{AD}$ and PSP. We tried to extract disease-specific features from these brains with AD and PSP for possible discrimination, based on the distribution of TAs and that of NFTs and immunohistochemistry for phosphorylated tau (AT8) [24], three-repeat (3R) and four-repeat (4R) tau [9]. This hybrid approach was quite successful in discriminating most, but

(c) The Author(s). 2019 Open Access This article is distributed under the terms of the Creative Commons Attribution 4.0 International License (http://creativecommons.org/licenses/by/4.0/), which permits unrestricted use, distribution, and 
Table 1 Demographic data on 5 patients with neuropathological diagnosis of AD and PSP.

\begin{tabular}{|c|c|c|c|c|c|c|c|c|c|c|}
\hline Case & $\begin{array}{l}\text { Age at } \\
\text { death }\end{array}$ & Sex & $\begin{array}{l}\text { Clinical } \\
\text { diagnosis }\end{array}$ & Dementia & Parkinsonism & $\begin{array}{l}\text { Duration of illness } \\
\text { (year) }\end{array}$ & $\begin{array}{l}\text { Neuropathological } \\
\text { findings }\end{array}$ & $\begin{array}{l}\text { Brain } \\
\text { weight (g) }\end{array}$ & $\begin{array}{l}\text { Braak NFT } \\
\text { stage }\end{array}$ & $\begin{array}{l}\text { Braak Amyloid } \\
\text { stage }\end{array}$ \\
\hline 1 & 86 & $F$ & $\mathrm{CHF}$ & - & - & N. A & $P S P, A D$ & 1205 & $\|$ & 0 \\
\hline 2 & 94 & M & possible DLB & + & + & 3 & $\begin{array}{l}\text { LB pathology, } \\
\text { PSP, AD, AGD }\end{array}$ & 1260 & III & A \\
\hline 3 & 81 & M & $A D$ & + & - & 9 & PSP, AD & 1230 & V & C \\
\hline 4 & 85 & M & pneumonia & N. A & N. A & N. A & $P S P, A D, C A A$ & 1395 & V & C \\
\hline 5 & 80 & M & possible PSP & + & + & 8 & $P S P, A D, A G D$ & 1210 & $\mathrm{VI}$ & C \\
\hline
\end{tabular}

$A D$ Alzheimer disease, $A G$ argyrophilic grain disease, CAA cerebral amyloid angiopathy, CHF congestive heart failure, DLB dementia with Lewy body, N. $A$ not available, NFT neurofibrillary tangle, PSP progressive supranuclear palsy

not all, of tau-positive lesions, suggesting that AD-type pathology and PSP-type pathology are independent without significant interaction even when coexistent in the same brain.

\section{Patients and methods}

Among one hundred and eighty autopsy cases archived at Laboratory of Structural Neuropathology, Tokyo Metropolitan Institute of Medical Science from 1999 to 2013, we picked up 5 cases (3 cases from Nitobe Memorial Nakano General Hospital and 2 cases from Yokufukai hospital) with comorbid pathologies with PSP [15] and AD [5]. Written consent from the patient's family was obtained at autopsy and this study was approved at the ethics review committee of the Tokyo Metropolitan Institute of Medical Science (authorization number 16-25).

Demographic data are summarized in Table 1. Median age was 85 years (range $80-94$ years) and male female ratio was $4: 1$. Brain weight was $1260 \mathrm{~g}$ on average (range 1205-1395 g), Braak NFT stage [5] had median V (range II-VI), and Braak Amyloid stage [5] had median B (range $0-C$ ).

Sixteen regions, selected to map PSP-specific or AD-specific lesions [2, 4-6, 14, 18, 22, 34, 40] throughout the brain include primary motor cortex (PC), putamen (PU), external segment of globus pallidus (GPE), internal segment of globus pallidus (GPI), subthalamic nucleus (STN), hippocampal formation (HF), substantia nigra

Table 2 Regional distribution of AT8 positive lesions.

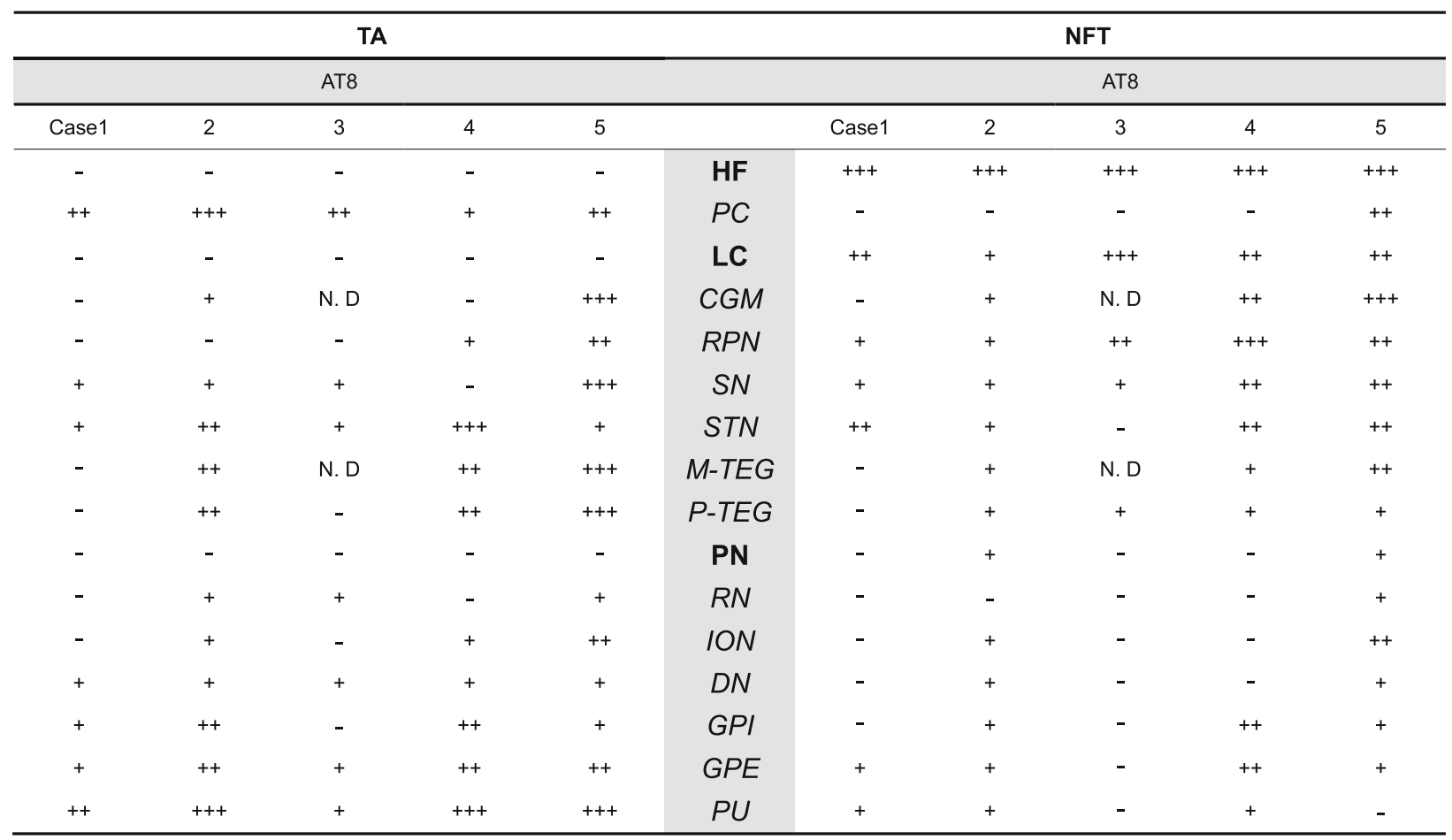

See Abbreviations after the text 


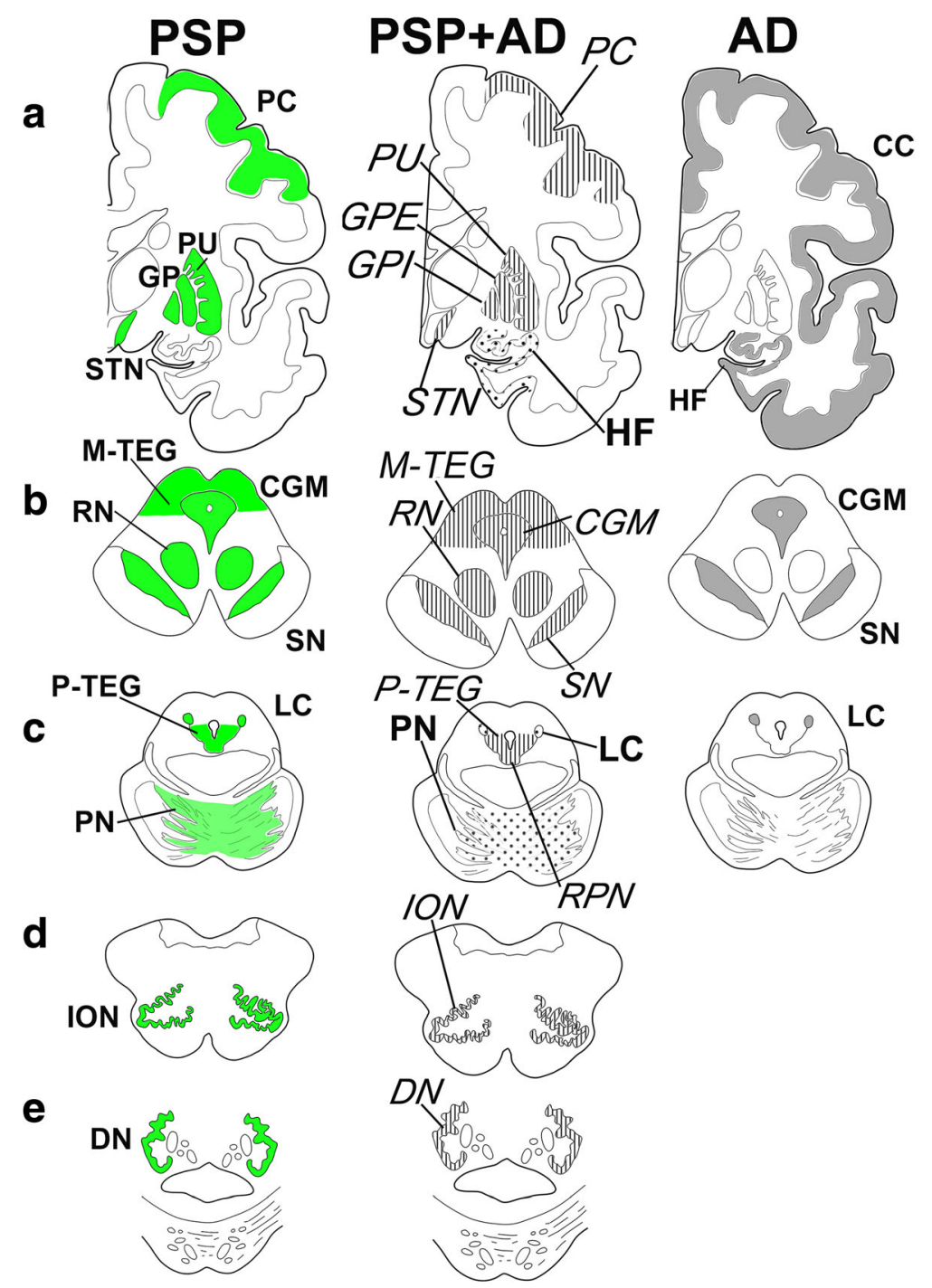

Fig. 1 Separation of tau-positive lesions into progressive supranuclear palsy (PSP) and Alzheimer disease (AD) based on their distribution and cytopathology. Tau-positive lesions of PSP shown in green (left column, a-e), typically include primary motor cortex (PC), putamen (PU), globus pallidus (GP), subthalamic nucleus (STN), central grey matter (CGM), substantia nigra (SN), red nucleus (RN), tegmentum (M-TEG), locus coeruleus $(\mathrm{LC})$, pontine nucleus $(\mathrm{PN})$ and tegmentum (P-TEG), inferior olivary nucleus (ION) and dentate nucleus (DN) in the cerebellum. Tau-positive lesions of Alzheimer disease (AD), shown in grey (right column A-C), are more restricted to CGM, SN and LC in the brainstem while more extended in the hippocampal formation (HF) and cerebral cortex (CC). In the five comorbid cases with PSP and AD (PSP + AD in the mid column), these taupositive lesions are partly overlapping. Regions with neurofibrillary tangles (NFTs) with tuft-shaped astrocytes (TAs) are labeled in italics, which replicate of PSP-type distribution (left column). Those with NFTs without TAs are labeled in Bold face (HF, LC and PN), which replicate AD-type distribution. Cerebral left hemisphere (coronal), b: Midbrain (axial), c: Pons (axial), d: Medulla oblongata (axial), e: Cerebellum.

$(\mathrm{SN})$, red nucleus $(\mathrm{RN})$, central grey matter (CGM), tegmentum of midbrain (mainly superior coliculli, excluding CGM;M-TEG), locus coeruleus (LC), raphe nucleus $(\mathrm{RPN})$, pontine nucleus (PN), tegmentum of pons (excluding LC and RPN;P-TEG), inferior olivary nucleus (ION) and dentate nucleus (DN). Six micron-thick sections were obtained from the formalin-fixed, paraffin-embedded blocks from these 16 regions. Deparaffinized sections were subjected to Hematoxylin-Eosin (HE) stain, Klüver-Barrera
(KB) stain, Gallyas silver impregnation and Campbell silver impregnation [36]. Isoform-specific antibodies directed against $3 \mathrm{R}$ or $4 \mathrm{R}$ tau [9] were used [37, 41]. Briefly, deparaffinized sections were treated for $15 \mathrm{~min}$ with $0.25 \%$ potassium permanganate $\left(\mathrm{KMnO}_{4}\right)$, for 3 min with $2 \%$ oxalic acid (OA), for 30 min with $>99 \%$ formic acid (FA) and for $20 \mathrm{~min}$ autoclaved at $121^{\circ} \mathrm{C}$ in $0.05 \mathrm{M}$ citrate buffer $[16,38]$. After intrinsic peroxidases were inactivated by $1 \%$ hydrogen peroxide $\left(\mathrm{H}_{2} \mathrm{O}_{2}\right)$ for 15 
Table 3 Regional distribution of 3R tau lesions and 4R tau lesions.

\begin{tabular}{|c|c|c|c|c|c|c|c|c|c|c|c|c|c|c|c|}
\hline \multicolumn{6}{|c|}{ TA } & \multicolumn{10}{|c|}{ NFT } \\
\hline \multicolumn{5}{|c|}{$4 R$} & & \multicolumn{5}{|c|}{$4 R$} & \multicolumn{5}{|c|}{$3 R$} \\
\hline Case1 & 2 & 3 & 4 & 5 & & Case1 & 2 & 3 & 4 & 5 & Case1 & 2 & 3 & 4 & 5 \\
\hline- & - & - & - & - & HF & + & + & ++ & ++ & +++ & +++ & +++ & +++ & +++ & +++ \\
\hline++ & +++ & ++ & + & ++ & $P C$ & - & - & - & - & ++ & - & - & - & - & ++ \\
\hline - & - & - & - & - & LC & + & + & + & + & ++ & ++ & + & +++ & ++ & ++ \\
\hline- & + & N. D & - & +++ & CGM & - & + & N. D & + & +++ & - & + & N. D & ++ & ++ \\
\hline- & - & - & + & ++ & $R P N$ & - & + & + & ++ & ++ & + & + & ++ & +++ & ++ \\
\hline+ & + & + & - & +++ & $S N$ & + & + & + & ++ & ++ & + & - & ++ & ++ & + \\
\hline+ & ++ & + & +++ & + & STN & ++ & + & - & ++ & ++ & + & - & - & + & - \\
\hline- & ++ & N. D & ++ & +++ & M-TEG & - & + & N. D & + & ++ & - & - & N. D & + & - \\
\hline- & ++ & - & ++ & +++ & P-TEG & - & + & - & + & + & - & - & + & + & + \\
\hline- & - & - & - & - & PN & - & + & - & - & + & - & - & - & - & - \\
\hline- & + & + & - & + & $R N$ & - & - & - & - & + & - & - & - & - & - \\
\hline- & + & - & + & ++ & $I O N$ & - & + & - & - & ++ & - & - & - & - & - \\
\hline+ & + & + & + & + & $D N$ & - & + & - & - & + & - & - & - & - & - \\
\hline+ & ++ & - & ++ & + & GPI & - & + & - & ++ & + & - & - & - & - & - \\
\hline+ & ++ & + & ++ & ++ & GPE & + & + & - & ++ & + & - & - & - & - & - \\
\hline++ & +++ & + & +++ & +++ & $P U$ & + & + & - & + & - & - & - & - & - & - \\
\hline
\end{tabular}

green: 4R tau region; yellow: $3+4 \mathrm{R}$ tau region

min, sections were incubated first with 5\% horse serum in $0.01 \mathrm{M}$ phosphate-buffered saline containing $0.03 \%$ polyoxyethylene (10) octylephenyl ether (Triton X-100, Wako, Tokyo, Japan; PBST). They were then incubated with either 3R tau-specific antibody (RD3 1:3000, Merck Millipore, Germany) or 4R tau-specific antibody (RD4 1:1000, Merck Millipore, Germany) diluted in the same buffer for 2 days at $4{ }^{\circ} \mathrm{C}$ [9]. They were then incubated with biotinylated secondary antibody against mouse IgG (1:1000, ABC Elite, Vector, Burlingame, CA) diluted in the same buffer for $2 \mathrm{~h}$ at room temperature. They were then incubated with avidin-biotin-peroxidase complex (1:1000, ABC Elite, Vector) for $1 \mathrm{~h}$ and visualized with diaminobenzidine and nickel ammonium chloride [38]. 4R or 3R immunoreactivity (IR) in each of 16 regions was separately evaluated semiquatitatively as none: 0 , mild ( 1 to 5 ): + ; moderate (6 to 10$)$ : ++ ; severe $(>10)$ : +++ , (lesions/visual field with $\times 20$ objective).

In some areas, where both $3 \mathrm{R}$ and $4 \mathrm{R}$ tau immunoreactivities (IR) were coexistent, double immunofluorolabeling was performed as described previously. The $6-\mu \mathrm{m}$ thick sections were deparaffinized for double immunofluorolabeling with antibodies against isoform-specific anti-4R tau antibody (rabbit polyclonal, Cosmo Bio Co, Tokyo, Japan), raised against amino acids 275-291 of human 4R tau, which is deaminated at N279 [8], and the anti-3R tau antibody (RD3) [9]. Sections were washed with PBST, blocked for $30 \mathrm{~min}$ in $5 \%$ normal goat serum $/ 0.05 \%$ sodium azide /PBS and incubated with the polyclonal anti-4R-tau antibody (1:3000) and RD3 (1:300), diluted in the blocking buffer at $4{ }^{\circ} \mathrm{C}$ for 4 days. To reduce autofluorescence of lipofuscin, sections were treated with Sudan Black B [28]. These primary antibodies were labeled with Alexa 488 conjugated with anti-rabbit IgG (Molecular Probes, Oregon, USA, 1:200) and Alexa 568 conjugated with anti-mouse IgG (Molecular Probes, Oregon, USA, 1:200), respectively, diluted in PBS with $0.03 \%$ Triton X-100 overnight in the dark. Sections were mounted with buffered glycerol containing $0.1 \% p$-phenylenediamine. Fluorescent signals were separately captured on a confocal system (Leica SP8; Leica Microsystems GmbH, Heidelberg, Germany) through a $63 \mathrm{x}$ objective (NA 1.45).

\section{Results}

Tau-positive lesions detected by AT8 were sorted into TA and NFT and semiquantitatively mapped in 16 regions as shown in Table 2. The distribution of tau-positive lesions in these 5 comorbid cases was schematized in Fig. 1 (center column). Comparison with that of PSP (Fig. 1, left column) and that of $\mathrm{AD}$ (Fig. 1, right column) demonstrated that the overall tau distribution in these five comorbid cases (Fig. 1, center column) was compatible with the summation of PSP (Fig. 1, left column) and AD (Fig. 1, right column). However, distribution of TA and that of NFT were not 


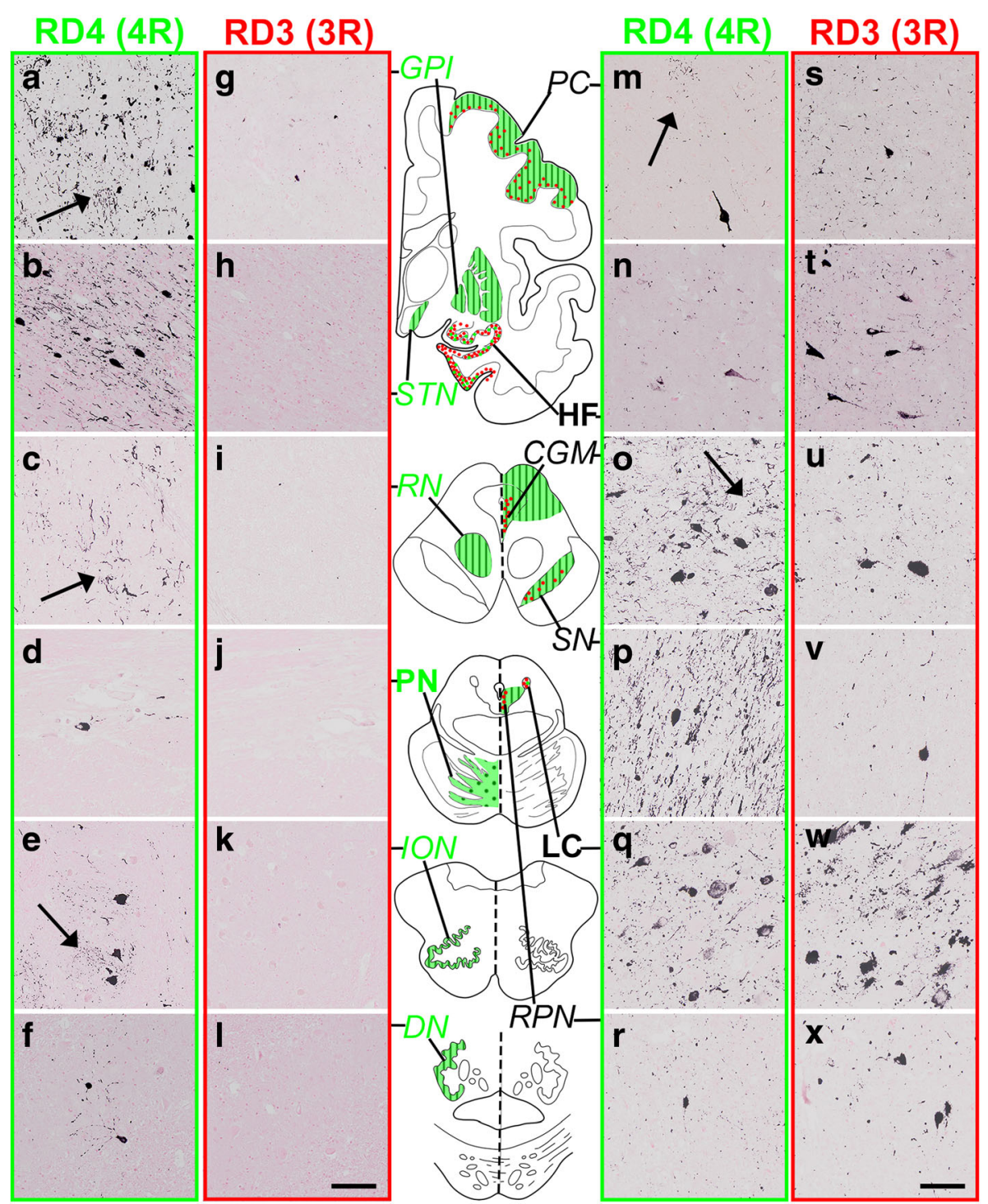

Fig. 2 Tau isoform-oriented mapping into four repeat only (4R+/3R-) regions for PSP type and three and four repeat (3R+/4R+) regions for $A D$ type distribution. Representative immunostaining for four-repeat (4R) tau (RD4, green) and three-repeat (3R) tau (RD3, red) in these twelve regions. The left set columns demonstrated 4R specific immunoreactivity (IR) without 3R IR in GPI (a, g), STN (b, h), RN (c, i), PN (d, j), ION (e, k) and DN (f, I), which replicates PSP-type distribution with glial involvement (arrows). In contrast, the right set columns demonstrated both $4 R$ and $3 R$ tau IR in PC (m, s), HF (n, t), CGM $(0, u)$, SN $(p, v), L C(q, w)$ and RPN (r, x), which replicates AD-type distribution. Glial lesions are exclusively positive for RD4 (arrows) but negative for RD3. Bars: $100 \mu \mathrm{m}$

similar. Both TA and NFT were detected in RN, ION, DN, GPI, GPE and PU (Tab. 2, italics), where tau-positive lesions are rare in pure AD. These regions containing both TA and NFT replicated the distribution of pure PSP (Fig. 1, left column), suggesting that these PSP-like tau lesions are not influenced by the copresence of $\mathrm{AD}$ pathology (Fig. 1, right column). However, both TA and NFT were found in PC, M-TEG, CGM, P-TEG and SN, where tau-positive lesions were found in pure AD as well (Tab. 2, Fig. 1).

To distinguish PSP-type and AD-type tau pathologies in these comorbid cases, tau-positive lesions, already sorted into NFT/TA (Tab. 2, Fig. 1), were further distinguished by $3 R$ and $4 R$ IR as shown with their relative amount in Table 3. Their immunohistochemical profiles are displayed in Fig. 2, where 4R-selective regions (Fig. 2, a-1, left: containing $4 R+/ 3 R-$ tau lesions) are contrasted with $4 R+3 R$ regions (Fig. 2, $\mathrm{m}-\mathrm{x}$ right: containing both $3 \mathrm{R}$ and $4 \mathrm{R}$-positive lesions). TAs were positive only for $4 \mathrm{R}$ tau and found in every area examined except for LC, HF, PN. 3R tau-positive TAs were absent even when 3R-positive NFT pathology was prominent as in LC (Fig. 2w).

$4 \mathrm{R}$-selective regions were GPI, RN, PN, ION, DN (Fig. 2 a-l, Table 3, regions in green box), where NFTs were positive only for $4 \mathrm{R}$ (Fig. 2 a-l, left), while STN 

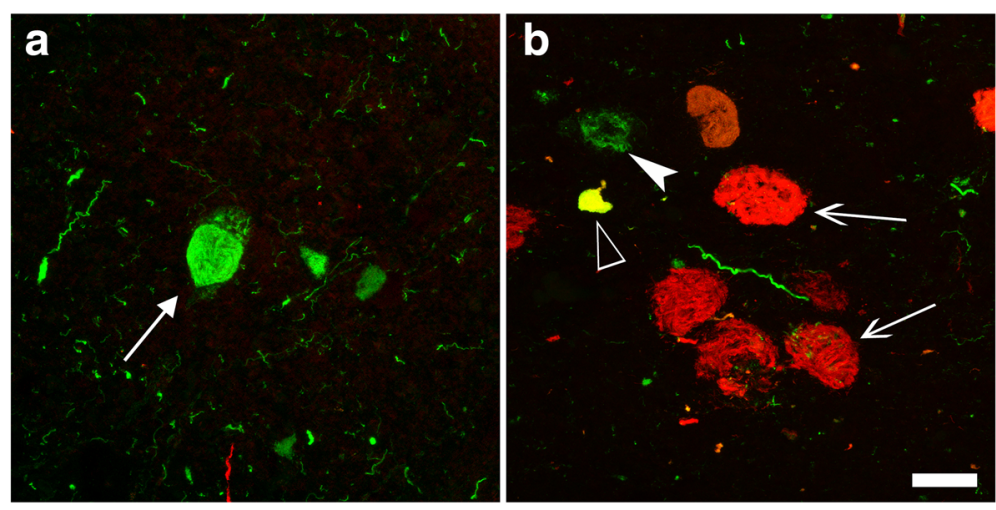

Fig. 3 Tau isoforms on NFTs are different from a region (SN) to another (CGM) even in the same brain (Case 5). Preferential 4R labeling (green) on NFT (arrow) in SN (a) represents PSP-type pathology. In CGM (b), NFTs are differently labeled for 4R (green, arrowhead), 3R (red, arrows) or both 4R. and 3R (yellow, empty arrow), representing AD-type pathology. Bar: $25 \mu \mathrm{m}$

contained a few NFTs positive for 3R. In contrast, NFTs positive for $4 R$ and $3 R$ tau were found in $4 R+3 R$ regions, including PC (Case 5 only), HF, CGM, SN, LC, RPN, M-TEG and P-TEG. (Fig. $2 \mathrm{~m}-\mathrm{x}$, Tab. 3, regions in yellow box). Among these $4 R+3 R$ regions, $H F$ and $L C$ were characterized by dominant 3R IR in NFTs over 4R IR and lack of 4R-positive glia (Tab. 3). NFTs in PC of case 5 (Braak NFT stage VI) exhibited dominant 3R IR over 4R IR. In other $4 \mathrm{R}+3 \mathrm{R}$ regions (CGM, RPN, SN, STN, M-TEG, P-TEG), 3R IR and 4R IR were comparable and tau-positive astrocytes were present in variable number. Double immunofluorolabeling (Fig. 3) for 4R tau (green) and 3R tau (red) demonstrated expected dominance of $4 \mathrm{R}$ tau (green) in $4 \mathrm{R}$-selective regions as $\mathrm{SN}$ (Fig. 3a). In contrast, the proportion of $3 \mathrm{R}$ tau (red) and $4 \mathrm{R}$ tau (green) was variable from a neuron to another (Fig. $3 b$ ) in $4 R+3 R$ regions as in CGM.

\section{Discussion}

Tau pathology of PSP-type and that of AD-type have been described separately [2, 4-6, 14, 22, 40], each representing separate entities. In this study with 5 cases harboring both PSP-type and AD-type tau pathology, we tried to discriminate PSP-type and AD-type pathologies in each brain along different criteria (distribution, participation of glial changes and tau isoforms). As initial description of PSP was based only on NFTs [31], comparison of their distribution provided a solid framework to distinguish pure PSP from pure AD (Fig. 1, Tab. 2). 4R-selective regions, characterized by the copresence of TA and NFT, both devoid of 3R tau IR (lower half of Tab. 3, in green box), include typical distribution of pure PSP (PN, RN, ION, DN, GPI, GPE and PU) [15], which are not severely affected in pure $\mathrm{AD}$. In contrast, involvement of $3 \mathrm{R}$ tau is seen in $\mathrm{HF}$ and $\mathrm{LC}$ in this series of cases with both disorders, which strongly suggests AD-type pathology $[17,21,33,37]$ rather than
PSP-pathology. This assumption is reinforced by the paucity of glial involvement in these regions as in pure $\mathrm{AD}$ cases [22] and by the predominance of 3R tau over $4 R$ tau (Tab. 3) [12]. Therefore, neuron-selective involvement with preferential $3 \mathrm{R}$ tau over $4 \mathrm{R}$ tau may represent AD-type pathology $[3,23]$ even in this comorbid series, which replicated AD-type distribution of NFT (HF, IC, LC, Fig. 4, right upper rectangle labeled AD). This is in contrast with PSP-type pathology with 4R-selective tau in both TA and NFTs (Fig. 4, left lower area in green) $[11,15]$, which replicated the PSP-type distribution. This operational sorting through our hybrid approach was powerful enough to distinguish origin of most of tau lesions into either AD-type or PSP-type (Fig. 4), suggesting that AD-type pathology and PSP-type pathology are independent even when these two processes are occurring in the same brain $[7,18,19,25,27]$.

This sharp distinction of AD-type pathology and PSP-type pathology in the same brain suggests that extension of AD-type pathology and PSP-type pathology are independently guided by distinct cytopathological mechanism along disease-specific patterns without crossover even if $4 \mathrm{R}$ tau is found in AD and PSP. If 4R-tau lesions extended transsynaptically (in a prion-like manner? [26]), AD-type and PSP-type lesions that are found together in some regions should also be found associated in their areas of projection. Because coexistent PSP-type pathology and AD-type pathology were distinct in the human brain, it is hard to explain how transsynaptic extension of $4 R$ tau, for example if any, exhibit different types of tau pathology in the same brain. Still, there remain some ambiguities in some regions such as STN, SN, M-TEG and P-TEG (area in broken line, Fig. 4 ), where very small amount of 3R-poitive NFTs are sometimes present. In these regions, 3R-positive NFTs (arrows in Fig. 3b, red) may be of AD-type even they are positive also for $4 \mathrm{R}$ tau (empty arrowhead in Fig. 3b, 


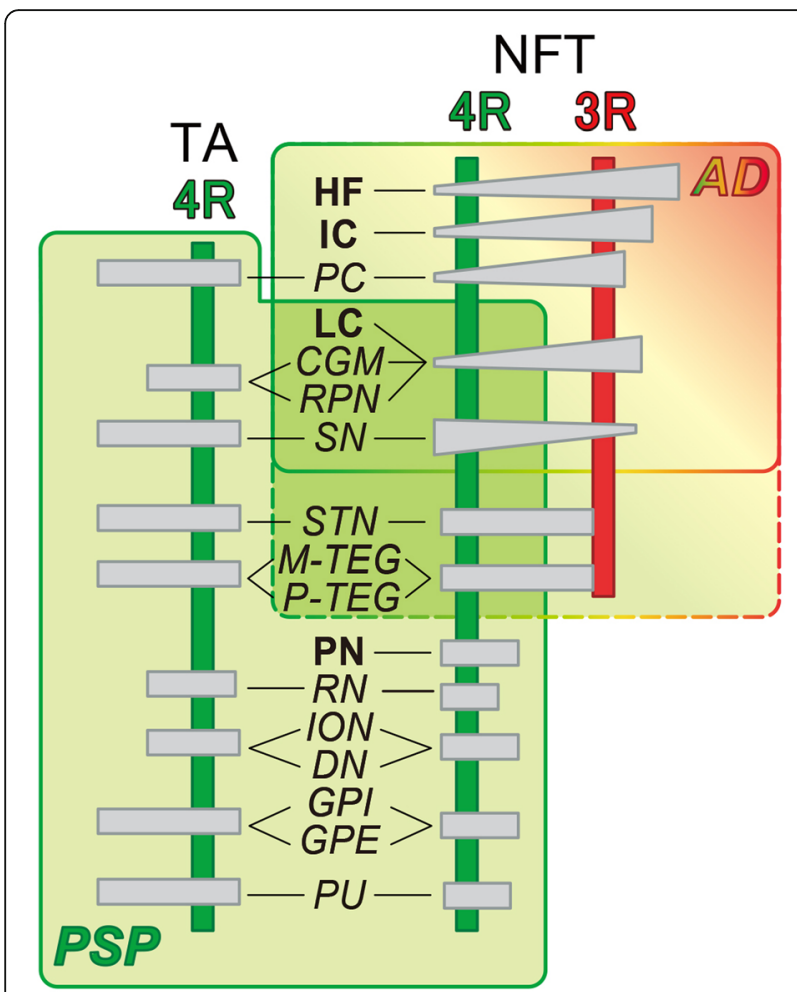

Fig. 4 How to differentiate the origin (PSP or AD) of each tau-positive lesion in comorbid brains with both PSP and AD pathology. Tau-positive lesions are sorted along regions indicated at the center column. Regions containing NFTs but not TAs are labeled in bold face (HF, IC: insular cortex, LC and PN). Other regions containing both NFTs and TAs are labeled in italics. Horizontal bars indicate relative quantity of TA and NFT in each region. IR to $4 R$ tau (green) and that to $3 R$ tau (red) are indicated by vertical lines. TAs are positive only for $4 R$ tau as indicated on the left half. In contrast, NFTs were sometimes positive for 3R tau in SN. 3R tau IR was more predominant in HF, IC, PC, LC, CGM and RPN, which replicates AD-like distribution partly shared with typical PSP. Rectangle in the right upper corner labeled as AD encompasses AD-type pathology in terms of distribution, further characterized by dominance of $3 R$ tau and lack of TAs. The left lower area labeled as PSP encompasses PSP-type pathology in terms of distribution with $4 R$ selectivity not only on TAs but also on NFTs. Although this chart provides concise and operational sorting of tau-positive lesions into AD or PSP origins, it is still difficult how to sort STN, M-TEG and P-TEG into PSP or AD (dotted area), because a few lesions exhibited $4 R$ tau $\mathbb{R}$

yellow) $[37,39]$. However, it is not yet clear whether 4R-positive neurons without $3 \mathrm{R}$ tau in these areas are of AD-type or PSP-type (Fig. 3b, arrowhead, green) [17, 37], because it is not yet possible to distinguish $4 \mathrm{R}$ tau of AD-type or of PSP-type. Indeed, 4R-tau lesions of AD and those of PSP similarly exhibit argyrophilia with Gallyas silver impregnation [36] and $4 R$ tau IR with 4R-specific antibodies such as monoclonal antibody RD4 [9] or polyclonal antibodies to 4R tau [8].

It has been reported that pretangle neurons are positive for $4 R$ tau but not for $3 R$ tau in both $A D$ and PSP $[17,37]$. However, this selective $4 R$ tau IR at early phase of tau deposition is gradually replaced with $3 R$ tau IR during evolution NFTs in AD brain [12,39]. This is in sharp contrast with PSP or CBD brain, where $4 R$ tau IR remains persistent without involvement of $3 R$ even after tau-positive fibrils are dense enough to form aggregated inclusions [20, 32]. Because currently available antibodies against $4 \mathrm{R}$ tau immunolabel tau deposits both in $\mathrm{AD}$ and in PSP/CBD brains [8], it remains to be clarified how 4R tau in AD brain and that in PSP brains are similar or different. However, if representation of $4 R$ tau deposits is disease-specific, it is expected that molecular species of $4 \mathrm{R}$ tau itself is disease-specific as well. For example, it has been reported that asparagine at residue 279 of $4 \mathrm{R}$ tau is deamidated to aspartate in AD brains [13], while this posttranslational change is not robust in PSP/CBD brains [8]. Immunoprobes that may detect such $\mathrm{AD}$-specific posttranslational changes may provide a straightforward strategy to demix PSP-type lesions and AD-type lesions in the same brain, in the same area or even within a single neuron at molecular level. If AD-type NFTs are characterized by paired helical filaments [29, 32] while PSP-type NFTs are characterized by straight fibrils [35] on electron microscopy, it will be exciting to examine how they are related to such biochemical differences, if any. Our hybrid approach to demix AD-type and PSP-type tau lesions may be corroborated by hybrid molecular demixing and electron microscopic studies, which will surely improve our mechanistic understanding of these diseases for more precise diagnosis and better management.

\section{Conclusions}

In human autopsied brains harboring both PSP-type and AD-type pathologies, tau-positive lesions were sorted based on their cell type (neuron vs glia), distribution and tau isoforms (3R vs $4 \mathrm{R}$ ). With this hybrid approach, we were successful in demixing PSP-type cytopathology (4R-selectivity in glia and neuron in PN, RN, ION, DN, GPI, GPE and PU) and AD-type cytopathology (3R and $4 \mathrm{R}$ in neuron in HF, insular cortex:IC and LC). However, this demixing is still incomplete because STN, SN, M-TEG and P-TEG contain tau lesions in neurons and glia are positive for $3 R$ and $4 R$. Further demixing may be possible if biochemical difference of $4 \mathrm{R}$ tau between PSP and $\mathrm{AD}$ is identified.

\section{Abbreviations}

3R: three-repeat; 4R: four-repeat; AD: Alzheimer disease; CC: cerebral cortex; CGM: central grey matter; DN: dentate nucleus; FA: formic acid; GPE: external segment of globus pallidus; GPI: internal segment of globus pallidus; $\mathrm{H}_{2} \mathrm{O}_{2}$ : hydrogen peroxide; HE: Hematoxylin-Eosin; HF: hippocampal formation; IC: insular cortex; ION: inferior olivary nucleus;

IR: immunoreactivity; KB: Klüver-Barrera; $\mathrm{KMnO}_{4}$ : potassium permanganate; LC: locus coeruleus; M-TEG: tegmentum of midbrain; NFT: neurofibrillary tangle; OA: oxalic acid; PBST: phosphate-buffered saline containing $0.03 \%$ polyoxyethylene (10) octylephenyl ether; PC: primary motor cortex;

PN: pontine nucleus; PSP: progressive supranuclear palsy; P-TEG: tegmentum 
of pons; PU: putamen; RN: red nucleus; RPN: raphe nucleus; SN: substantia nigra; STN: subthalamic nucleus; TA: tuft-shaped astrocyte

\section{Acknowledgements}

Not applicable.

\section{Funding}

This work was supported by Grant-in-Aid for JSPS KAKENHI Grant Numbers $17 \mathrm{H} 03555$ (TU), 16 K14572 (TU).

\section{Authors' contribution}

Study design: ME, YI, TU; Autopsy and neuropathological analyses: ME, Yl, $\mathrm{KH}$ TU; Immunohistochemical analyses and mapping: ME, MU, AN, SK, TU; Initial drafting: ME, SK; Final drafting: KH, SK, TU. All authors have read and approved the final manuscript.

\section{Ethics approval and consent to participate}

Written consent for autopsy and subsequent use of autopsied samples for research was obtained from the next of kin of each patient. The ethics committee of Tokyo Metropolitan Institute of Medical Science approved this study. (\#16-25).

\section{Competing interests}

The authors declare that they have no interests.

\section{Publisher's Note}

Springer Nature remains neutral with regard to jurisdictional claims in published maps and institutional affiliations.

\section{Author details}

${ }^{1}$ Laboratory of Structural Neuropathology, Tokyo Metropolitan Institute of Medical Science, Tokyo, Japan. ${ }^{2}$ Department of Neurology, Nihon University, School of Medicine, Tokyo, Japan. ${ }^{3}$ Department of Neurology, Yokufukai Hospital, Tokyo, Japan. ${ }^{4}$ Department of Pathology, Nitobe-Memorial Nakano General Hospital, Tokyo, Japan. ${ }^{5}$ Neurology Clinic with Neuromorphomics laboratory, Nitobe-Memorial Nakano General Hospital, Tokyo, Japan.

Received: 11 February 2019 Accepted: 22 March 2019

Published online: 06 May 2019

\section{References}

1. Alzheimer A, Stelzmann RA, Schnitzlein HN, Murtagh FR (1995) An English translation of Alzheimer's 1907 paper, "Uber eine eigenartige Erkankung der Hirnrinde". Clin Anat 8:429-431. https://doi.org/10.1002/ca.980080612

2. Armstrong RA, Lantos PL, Cairns NJ (2007) Progressive supranuclear palsy (PSP): a quantitative study of the pathological changes in cortical and subcortical regions of eight cases. J Neural Transm (Vienna) 114:1569-1577. https://doi.org/10.1007/s00702-007-0796-3

3. Bancher C, Lassmann H, Budka H, Grundke-lqbal I, lqbal K, Wiche G, et al. (1987) Neurofibrillary tangles in Alzheimer's disease and progressive supranuclear palsy: antigenic similarities and differences. Microtubuleassociated protein tau antigenicity is prominent in all types of tangles. Acta Neuropathol 74:39-46

4. Braak E, Braak H, Mandelkow EM (1994) A sequence of cytoskeleton changes related to the formation of neurofibrillary tangles and neuropil threads. Acta Neuropathol 87:554-567

5. Braak H, Braak E (1991) Neuropathological stageing of Alzheimer-related changes. Acta Neuropathol 82:239-259

6. Braak H, Del Tredici K (2011) The pathological process underlying Alzheimer's disease in individuals under thirty. Acta Neuropathol 121:171-181. https://doi.org/10.1007/s00401-010-0789-4

7. Cruz-Sanchez FF, Rossi ML, Cardozo A, Deacon P, Tolosa E (1992) Clinical and pathological study of two patients with progressive supranuclear palsy and Alzheimer's changes. Antigenic determinants that distinguish cortical and subcortical neurofibrillary tangles. Neurosci Lett 136:43-46

8. Dan A, Takahashi M, Masuda-Suzukake M, Kametani F, Nonaka T, Kondo et al. (2013) Extensive deamidation at asparagine residue 279 accounts for weak immunoreactivity of tau with RD4 antibody in Alzheimer's disease brain. Acta Neuropathol Commun 1:54. https://doi.org/10.1186/2051-5960-1-54

9. de Silva R, Lashley T, Gibb G, Hanger D, Hope A, Reid et al. (2003) Pathological inclusion bodies in tauopathies contain distinct complements of tau with three or four microtubule-binding repeat domains as demonstrated by new specific monoclonal antibodies. Neuropathol Appl Neurobiol 29:288-302

10. Evidente VG, Adler CH, Sabbagh MN, Connor DJ, Hentz JG, Caviness JN et al. (2011) Neuropathological findings of PSP in the elderly without clinical PSP: possible incidental PSP? Parkinsonism Relat Disord 17:365-371. https:// doi.org/10.1016/j.parkreldis.2011.02.017

11. Flament S, Delacourte A, Verny M, Hauw JJ, Javoy-Agid F (1991) Abnormal tau proteins in progressive supranuclear palsy. Similarities and differences with the neurofibrillary degeneration of the Alzheimer type. Acta Neuropathol 81:591-596

12. Hara M, Hirokawa K, Kamei S, Uchihara T (2013) Isoform transition from fourrepeat to three-repeat tau underlies dendrosomatic and regional progression of neurofibrillary pathology. Acta Neuropathol 125:565-579. https://doi.org/10.1007/s00401-013-1097-6

13. Hasegawa M, Morishima-Kawashima M, Takio K, Suzuki M, Titani K, Ihara Y (1992) Protein sequence and mass spectrometric analyses of tau in the Alzheimer's disease brain. J Biol Chem 267:17047-17054

14. Hattori M, Hashizume $Y$, Yoshida M, Iwasaki Y, Hishikawa N, Ueda R, et al. (2003) Distribution of astrocytic plaques in the corticobasal degeneration brain and comparison with tuft-shaped astrocytes in the progressive supranuclear palsy brain. Acta Neuropathol 106:143-149. https://doi.org/10.1007/s00401-003-0711-4

15. Hauw JJ, Daniel SE, Dickson D, Horoupian DS, Jellinger K, Lantos et al. (1994) Preliminary NINDS neuropathologic criteria for Steele-RichardsonOlszewski syndrome (progressive supranuclear palsy). Neurology 44: 2015-2019

16. Ichihara K, Uchihara T, Nakamura A, Suzuki Y, Mizutani T (2009) Selective deposition of 4-repeat tau in cerebral infarcts. J Neuropathol Exp Neurol 68: 1029-1036. https://doi.org/10.1097/NEN.0b013e3181b56bf4

17. Iseki E, Yamamoto R, Murayama N, Minegishi M, Togo T, Katsuse O, et al. (2006) Immunohistochemical investigation of neurofibrillary tangles and their tau isoforms in brains of limbic neurofibrillary tangle dementia. Neurosci Lett 405:29-33. https://doi.org/10.1016/j.neulet.2006.06.036

18. Ito K, Arai K, Yoshiyama Y, Kashiwado K, Sakakibara Y, Hattori T (2008) Astrocytic tau pathology positively correlates with neurofibrillary tangle density in progressive supranuclear palsy. Acta Neuropathol 115:623-628. https://doi.org/10.1007/s00401-008-0378-y

19. Keith-Rokosh J, Ang LC (2008) Progressive supranuclear palsy: a review of co-existing neurodegeneration. Can J Neurol Sci 35:602-608

20. Kumar $\mathrm{H}$, Udgaonkar JB (2018) Mechanistic and structural origins of the asymmetric barrier to prion-like cross-seeding between tau-3R and tau-4R. J Mol Biol 430:53045312. https:/doi.org/10.1016/j.jmb.2018.09.010

21. Lace G, Savva GM, Forster G, de Silva R, Brayne C, Matthews FE, Barclay JJ, Dakin L, Ince PG, al WSB (2009) Hippocampal tau pathology is related to neuroanatomical connections: an ageing population-based study. Brain 132 1324-1334. https://doi.org/10.1093/brain/awp059

22. Matsusaka H, Ikeda K, Akiyama H, Arai T, Inoue M, Yagishita S (1998) Astrocytic pathology in progressive supranuclear palsy: significance for neuropathological diagnosis. Acta Neuropathol 96:248-252

23. McKhann G, Drachman D, Folstein M, Katzman R, Price D, Stadlan EM (1984) Clinical diagnosis of Alzheimer's disease: report of the NINCDS-ADRDA work group under the auspices of Department of Health and Human Services Task Force on Alzheimer's disease. Neurology 34:939-944

24. Mercken $M$, Vandermeeren $M$, Lubke U, Six J, Boons J, Van de Voorde A, et al. (1992) Monoclonal antibodies with selective specificity for Alzheimer tau are directed against phosphatase-sensitive epitopes. Acta Neuropathol 84:265-272

25. Oshima K, Dickson DW (2009) Cortical Alzheimer type pathology does not influence tau pathology in progressive supranuclear palsy. Int J Clin Exp Pathol 2:399-406

26. Prusiner SB (1984) Some speculations about prions, amyloid, and Alzheimer's disease. N Engl J Med 310:661-663. https://doi.org/10.1056/ NEJM198403083101021

27. Sakamoto R, Tsuchiya K, Yoshida R, Itoh Y, Furuta N, Kosuga A, et al. (2009) Progressive supranuclear palsy combined with Alzheimer's disease: a clinicopathological study of two autopsy cases. Neuropathology 29:219-229. https://doi.org/10.1111/j.1440-1789.2008.00968.x

28. Schnell SA, Staines WA, Wessendorf MW (1999) Reduction of lipofuscin-like autofluorescence in fluorescently labeled tissue. J Histochem Cytochem 47: 719-730. https://doi.org/10.1177/002215549904700601 
29. Shibayama H, Kitoh J (1978) Electron microscopic structure of the Alzheimer's neurofibrillary changes in case of atypical senile dementia. Acta Neuropathol 41:229-234

30. Sperling RA, Aisen PS, Beckett LA, Bennett DA, Craft S, Fagan AM, et al. (2011) Toward defining the preclinical stages of Alzheimer's disease: recommendations from the National Institute on Aging-Alzheimer's Association workgroups on diagnostic guidelines for Alzheimer's disease. Alzheimers Dement 7: 280-292. https:/doi.org/10.1016/j.jalz:2011.03.003

31. Steele JC, Richardson JC, Olszewski J (1964) Progressive supranuclear palsy. A heterogeneous degeneration involving the brain stem, basal ganglia and cerebellum with vertical gaze and pseudobulbar palsy, nuchal dystonia and dementia. Arch Neurol 10:333-359

32. Tatsumi S, Uchihara T, Aiba I, Iwasaki Y, Mimuro M, Takahashi R, et al. (2014) Ultrastructural differences in pretangles between Alzheimer disease and corticobasal degeneration revealed by comparative light and electron microscopy. Acta Neuropathol Commun 2:161. https://doi.org/10.1186/ s40478-014-0161-3

33. Togo T, Akiyama H, Iseki E, Uchikado H, Kondo H, Ikeda K, et al. (2004) Immunohistochemical study of tau accumulation in early stages of Alzheimer-type neurofibrillary lesions. Acta Neuropathol 107:504-508. https://doi.org/10.1007/s00401-004-0842-2

34. Togo T, Dickson DW (2002) Tau accumulation in astrocytes in progressive supranuclear palsy is a degenerative rather than a reactive process. Acta Neuropathol 104:398-402. https://doi.org/10. 1007/s00401-002-0569-x

35. Tomonaga M (1977) Ultrastructure of neurofibrillary tangles in progressive supranuclear palsy. Acta Neuropathol 37:177-181

36. Uchihara $\mathrm{T}$ (2007) Silver diagnosis in neuropathology: principles, practice and revised interpretation. Acta Neuropathol 113:483-499. https:/doi.org/ 10.1007/s00401-007-0200-2

37. Uchihara T, Hara M, Nakamura A, Hirokawa K (2012) Tangle evolution linked to differential 3- and 4-repeat tau isoform deposition: a double immunofluorolabeling study using two monoclonal antibodies. Histochem Cell Biol 137:261-267. https://doi.org/10.1007/s00418-011-0891-2

38. Uchihara T, Nakamura A, Shibuya K, Yagishita S (2011) Specific detection of pathological three-repeat tau after pretreatment with potassium permanganate and oxalic acid in PSP/CBD brains. Brain Pathol 21:180-188. https://doi.org/10.1111/j.1750-3639.2010.00433.x

39. Uematsu M, Nakamura A, Ebashi M, Hirokawa K, Takahashi R, Uchihara T (2018) Brainstem tau pathology in Alzheimer's disease is characterized by increase of three repeat tau and independent of amyloid beta. Acta Neuropathol Commun 6:1. https://doi.org/10.1186/s40478-017-0501-1

40. Verny M, Duyckaerts C, Agid Y, Hauw JJ (1996) The significance of cortical pathology in progressive supranuclear palsy. Clinico-pathological data in 10 cases. Brain 119 (Pt4) 4:1123-1136

41. Yoshida M (2006) Cellular tau pathology and immunohistochemical study of tau isoforms in sporadic tauopathies. Neuropathology 26:457-470. https:// doi.org/10.1111/j.1440-1789.2006.00743.x

Ready to submit your research? Choose BMC and benefit from:

- fast, convenient online submission

- thorough peer review by experienced researchers in your field

- rapid publication on acceptance

- support for research data, including large and complex data types

- gold Open Access which fosters wider collaboration and increased citations

- maximum visibility for your research: over $100 \mathrm{M}$ website views per year

At BMC, research is always in progress.

Learn more biomedcentral.com/submissions 